\title{
Phase coherence and superfluid-insulator transition in a disordered Bose-Einstein condensate
}

\author{
Yong P. Chen, ${ }^{1,2, *}$ J. Hitchcock, ${ }^{1}$ D. Dries, ${ }^{1}$ M. Junker, ${ }^{1}$ C. Welford, ${ }^{1}$ and R. G. Hulet ${ }^{1}$ \\ ${ }^{1}$ Department of Physics and Astronomy and Rice Quantum Institute, Rice University, 6100 Main Street, Houston, Texas 77005, USA \\ ${ }^{2}$ The Richard E. Smalley Institute for Nanoscale Science and Technology, Rice University, 6100 Main Street, Houston, Texas 77005, USA
}

(Received 26 October 2007; published 31 March 2008)

\begin{abstract}
We have studied the effects of a disordered optical potential on the transport and phase coherence of a Bose-Einstein condensate (BEC) of ${ }^{7} \mathrm{Li}$ atoms. At moderate disorder strengths $\left(V_{D}\right)$, we observe inhibited transport and damping of dipole excitations, while in time-of-flight images, random but reproducible interference patterns are observed. In situ images reveal that the appearance of interference is correlated with density modulation, without complete fragmentation. At higher $V_{D}$, the interference contrast diminishes as the BEC fragments into multiple pieces with little phase coherence.
\end{abstract}

DOI: 10.1103/PhysRevA.77.033632

PACS number(s): 03.75.Hh, 03.75.Kk, 64.60.Cn

The behavior of a superfluid or a superconductor in the presence of disorder is of fundamental interest. A superfluid can flow without friction around obstacles, and a superconductor can have zero resistance despite material defects. On the other hand, disorder is able to localize particles, resulting in an insulating state [1]. Experimentally, disorder-induced superfluid/superconductor to insulator transitions (SIT) have been probed in many systems, including superfluid helium in porous media [2], thin-film and granular superconductors $[3,4]$, and random Josephson junction arrays [5]. While many believe that such a SIT is a quantum phase transition driven by quantum fluctuations, it remains a central task to understand exactly how the superfluid/superconducting order parameter, which consists both an amplitude and a phase, may be destroyed with increasing disorder. Numerous fundamental questions remain, such as the nature of the insulator, the fate of phase coherence throughout the transition, and the possibility of intermediate metallic phases $[3,6,7]$.

Cold atoms, with their intrinsic cleanliness coupled with remarkable controllability of physical parameters, have emerged as exceptional systems to study various condensed matter problems. Recently, several experiments [8-11] have studied ${ }^{87} \mathrm{Rb}$ condensates in random optical potentials and observed, for example, damping of collective excitations [8] and inhibition of expansion [9-11] due to disorder. Another experiment [12] has examined a Bose-Einstein condensate (BEC) in an incommensurate (quasirandom) optical lattice in order to investigate a possible "Bose-glass" phase [13]. Experiments with disordered atomic quantum gases may provide unique insights into disordered quantum systems and may uncover a rich variety of quantum phases [14].

Here we report experiments on a BEC of interacting ${ }^{7} \mathrm{Li}$ atoms subject to a well-controlled disordered potential. While we corroborate previous transport measurements [8-11], we have also probed the ground-state density distribution and phase coherence of the disordered BEC by performing both in situ and time-of-flight (TOF) imaging. While disorder inhibits transport of the BEC, reproducible TOF interference patterns are observed for intermediate disorder

\footnotetext{
*Current address: Purdue University, 525 Northwestern Ave., West Lafayette, IN 47907, USA.
}

strengths $V_{D}$, reflecting an underlying phase coherence in the disordered BEC. At stronger $V_{D}$, the interference contrast diminishes as the BEC fragments into a "granular" condensate, which is expected to have no phase coherence.

We create an optically trapped $\mathrm{BEC}$ of ${ }^{7} \mathrm{Li}$ atoms in the $m_{J}=-1 / 2, m_{I}=3 / 2$ state at a bias magnetic field $B \simeq 720 \mathrm{G}$ using procedures essentially similar to those described in [15]. The bias field tunes the scattering length $a_{s}$ via a Feshbach resonance to a large positive value $\left(\sim 200 a_{0}\right.$, where $a_{0}$ is the Bohr radius), enabling efficient evaporative cooling $[15,16]$. The optical dipole trap is produced by focusing a $1030 \mathrm{~nm}$ Yb:YAG laser beam to a Gaussian $1 / e^{2}$ intensity radius of $34 \mu \mathrm{m}$. In the following, we label the axial direction (along which the optical trap beam propagates) as $z$ and the two radial directions as $x$ and $y$. At the final trap depth, the radial trap frequencies are $\omega_{x}=\omega_{y} \simeq 2 \pi \times 180 \mathrm{~Hz}$ and the axial trap frequency is $\omega_{z} \simeq 2 \pi \times 3.6 \mathrm{~Hz}$. Part of the axial confinement is contributed by a residual curvature from the bias magnetic field. At this trap depth, the BEC contains $\sim 5 \times 10^{5}$ atoms with no discernible thermal component. The corresponding chemical potential $\mu \approx h \times 1 \mathrm{kHz}$, where $h$ is Planck's constant.

To create the disordered potential, we pass another beam, derived from the same laser as used for the optical trap, through a diffusive plate. This results in laser speckle [17] similar to that used in $[8,10]$. This beam propagates along the $x$ direction. Because of the optical dipole force, the speckle's spatially varying intensity pattern gives rise to a disordered potential $V(x, y, z)$ for the atoms. Figure 1(a) shows a twodimensional (2D) cross-section $V(0, y, z)$ as imaged by a camera, while the main panel shows an axial cut $V(z)$ $=V(0,0, z)$ of the disordered potential through the center $(x$ $=y=0)$ of the atomic cloud. The disorder strength $V_{D}$ is proportional to the intensity of the speckle laser and is defined as the standard deviation of the mean of $V(z)$. The disorder correlation length along the $z$ direction, $\sigma_{z}$, is defined by fitting the autocorrelation function $\langle V(z) V(z+\Delta z)\rangle$ to $e^{-2 \Delta z^{2} / \sigma_{z}^{2}}$ [Fig. 1(b)]; we find $\sigma_{z} \simeq 15 \mu \mathrm{m}$, which is much greater than the condensate healing length of $\sim 1 \mu \mathrm{m}$. Since $\sigma_{x} \gg \sigma_{y}=\sigma_{z}[10,17]$ and the radial Thomas-Fermi radius of the BEC is less than $10 \mu \mathrm{m}$, to a good approximation the $\mathrm{BEC}$ is subject to a $1 \mathrm{D}$ disordered potential $V(z)=V(0,0, z)$ along its axial direction. 


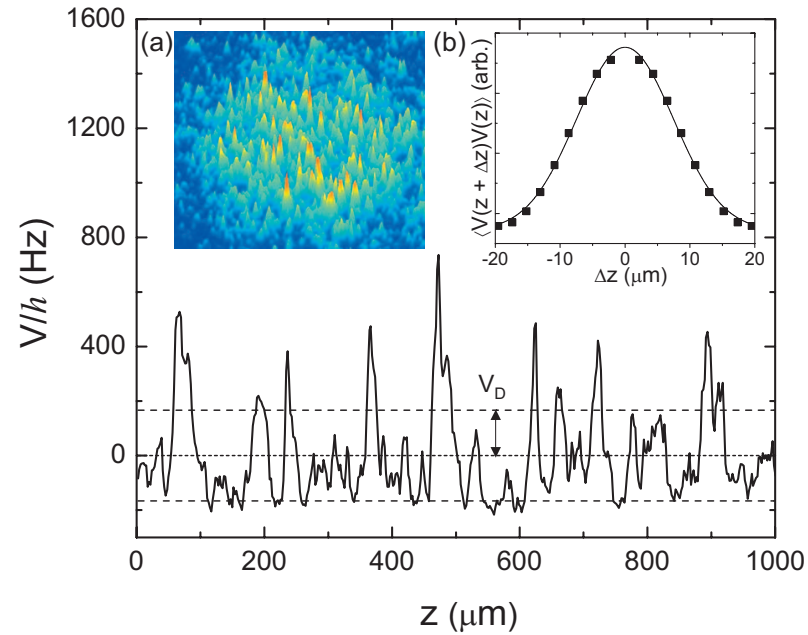

FIG. 1. (Color) Typical 1D disordered potential $V(z)$. (a) Falsecolor surface plot of the disordered speckle potential $V(y, z)$. The pixel size corresponds to $2.2 \mu \mathrm{m}$ in the plane of the atoms. (b) Autocorrelation function of $V(z)$. The line is a fit to $e^{-2 \Delta z^{2} / \sigma_{z}^{2}}$, within a scaling factor. As expected for fully developed speckle, the intensity follows a negative exponential probability distribution.

After producing a pure BEC (to within our resolution), the disordered potential is ramped on in $\sim 100 \mathrm{~ms}[18]$ to a specific value of $V_{D}$, which we express in terms of $\mu$. The BEC is then held in the disordered and trap potentials while various experiments are performed, as described below. The final state is probed by absorption imaging (also along the $x$ direction) of the atomic cloud released from the disordered and optical trap potentials or by in situ phase-contrast imaging [19].

We first describe data characterizing the transport properties of the disordered BEC. In one type of measurement, we slowly ramp on a magnetic field gradient along the $z$ direction, which offsets the center of the harmonic trap from $z$ $=0$ to $z=-d$. The disordered potential is kept stationary during the trap offset. As shown in Figs. 2(a) and 2(b), without disorder the center of the atomic cloud follows the trap. For intermediate disorder strength the cloud lags behind the new trap center and is stretched, as shown in Fig. 2(c). For stronger disorder, as shown in Fig. 2(d), the cloud is pinned at its initial position and does not respond to the offset. We plot the cloud center as a function of $V_{D}$ for four different offset distances $d$ in Fig. 2. The cloud is pinned and does not respond to trap offset for $V_{D} \gtrsim 0.8 \mu$, within our experimental time scale and uncertainty.

We have also studied the effect of disorder on the dipole oscillation of the trapped BEC (Fig. 3). In contrast to the slow offset experiment discussed above, here the harmonic trap center is abruptly (2 ms) offset and the cloud evolves in the presence of the shifted trap and stationary disorder for a variable time before being released for imaging. Without disorder, the condensate undergoes undamped dipole oscillations in the trap with frequency $\omega_{z}$. Even disorder as small as $V_{D} \sim 0.1 \mu$, however, damps these oscillations. Increasing the disorder strength to $V_{D} \gtrsim 0.4 \mu$ causes the dipole motion to be overdamped, until when $V_{D} \gtrsim \mu$, the cloud becomes pinned at its initial position to within our experimental resolution.

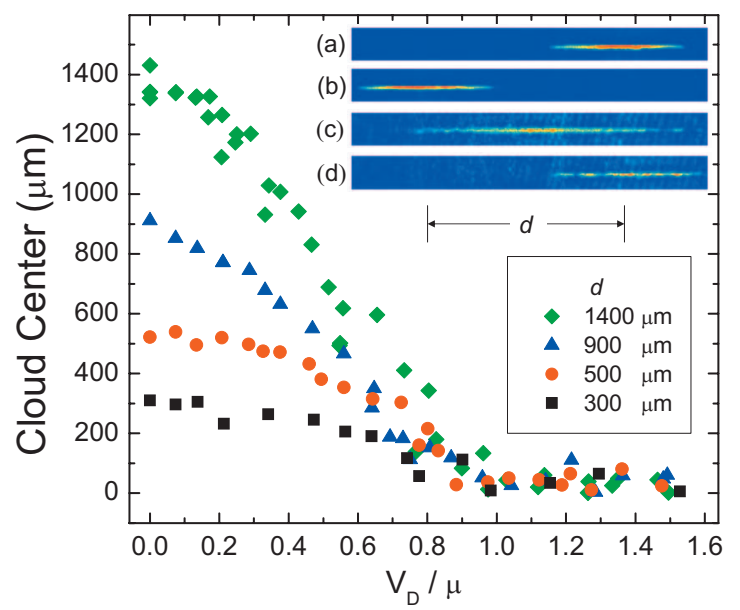

FIG. 2. (Color) Effect of disorder on slow transport of the BEC. The trap center is offset to the left for a distance $d$ over a time interval of $700 \mathrm{~ms}$. (a) Initial state of the BEC, without disorder or offset. The center of this initial cloud defines the reference position "0." (b)-(d) Result of offsetting the trap by $d \approx 1400 \mu \mathrm{m}$, with the cloud subjected to a disordered potential of various $V_{D}$ : (b) $V_{D}=0$, (c) $V_{D} \approx 0.3 \mu$, and (d) $V_{D} \approx \mu$. Images (a)-(d) are obtained by absorption imaging taken with a 1-ms TOF after the cloud is abruptly released from the disordered and optical trap potentials. Main panel shows the center by weight of the cloud as a function of $V_{D}$, for several offsets $d$. $V_{D}$ and $\mu$ have uncertainties of $\sim 20 \%$.

In addition to transport measurements, we have performed in situ [20] and TOF imaging on the disordered BEC, yielding information on both its density and phase. Figures 4(a) and 4(b) show in situ and corresponding TOF images taken 8 ms after releasing the cloud. For intermediate $V_{D}$ [Figs. $4(\mathrm{~b})$ and 4(h)] striking random fringes, which we interpret as matter wave interference, develop after sufficiently long TOF expansion of the BEC following release from the optical potentials. We note that at such $V_{D}$, the corresponding in situ

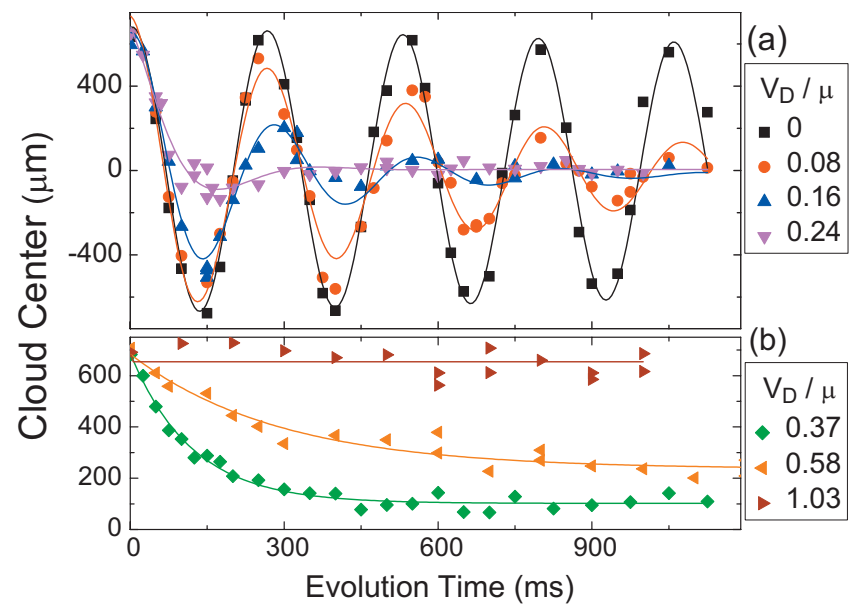

FIG. 3. (Color) Effect of disorder on dipole excitation of the condensate. Data show the cloud center as a function of time following a sudden axial offset of the trap for various disorder strengths $V_{D}$ (shown in two separate panels for clarity). The lines are fits to damped dipole oscillations. 

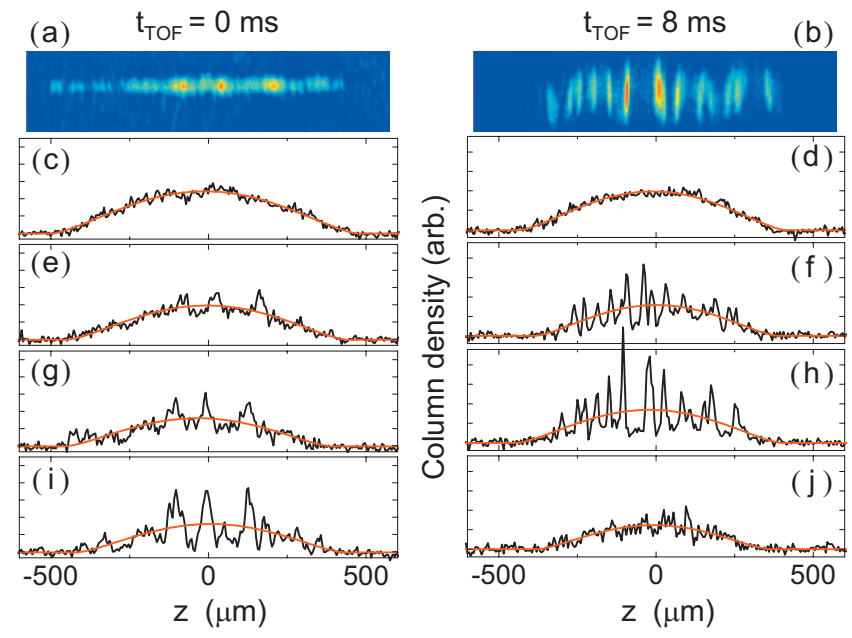

FIG. 4. (Color) In situ profiles and matter wave interference. (a) In situ image for $V_{D} \approx 0.5 \mu$ and (b) TOF image for $V_{D} \approx 0.5 \mu$. The TOF 2D image uses a different color scale from the one used for the in situ 2D image, and the displayed aspect ratio of the in situ images is reduced by $50 \%$ in order to enhance the transverse size for visibility. (c)-(j) In situ column-density profiles on left and corresponding TOF profiles on right: (c),(d) $V_{D}=0$; (e),(f) $V_{D}$ $\approx 0.3 \mu ;(\mathrm{g}),(\mathrm{h}) V_{D} \approx 0.5 \mu$; and (i),(j) $V_{D} \approx 1.0 \mu$. Solid red lines are fits to Thomas-Fermi distributions. $\omega_{z} \simeq 2 \pi \times 2.8 \mathrm{~Hz}$ for data shown in Figs. 4 and 5.

images [Figs. 4(a) and 4(g)] are consistent with the cloud still being connected. By increasing $V_{D}$ above $\mu$, the disordered BEC becomes fragmented as shown in the in situ profile, Fig. 4(i), and the fringe contrast observed in TOF diminishes, as shown in Fig. 4(j). To quantify the dependence of the TOF fringe pattern on $V_{D}$, we plot both the TOF and in situ contrast versus $V_{D} / \mu$ in Fig. 5. The TOF fringe contrast peaks at $V_{D} \simeq 0.5 \mu$, while the contrast in the in situ images increases up to the highest $V_{D}$ investigated.

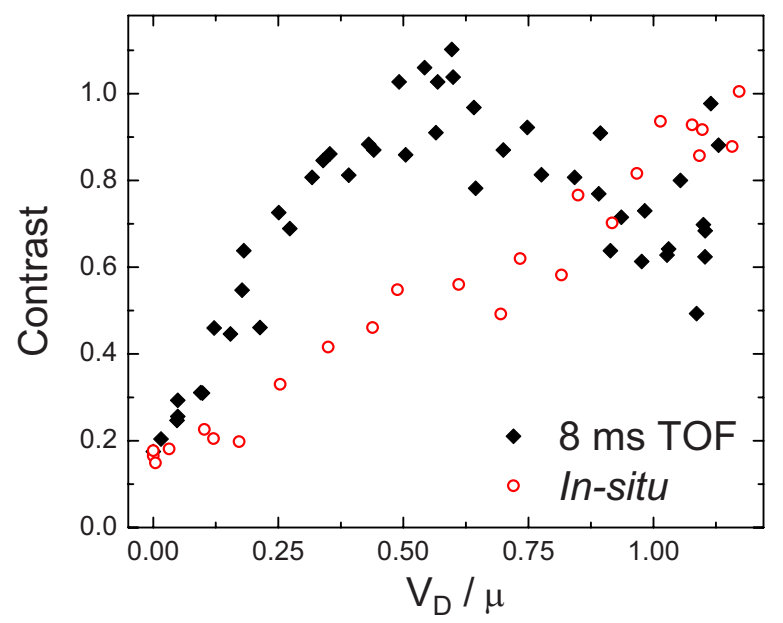

FIG. 5. (Color online) Contrast of the matter wave interference pattern in 8-ms TOF images (solid diamonds) and contrast of in situ density fluctuations (open circles) as a function of $V_{D} / \mu$. The contrast is extracted from the central axial cut of the column density as its mean deviation from the Thomas-Fermi fit normalized by the peak value of the fit.
Similar, but less well resolved fringes in TOF images have been reported previously $[8,11]$. Our highly elongated BEC facilitates a systematic and quantitative study of the fringes. We find that the positions of these irregularly spaced fringes are reproducible in repeated measurements, even with different holding times of the BEC in the disordered potential. During preparation of this paper, we became aware of a closely related paper [21], which also reports reproducible fringes in TOF images of a disordered BEC. The reproducibility suggests that these fringes are unlikely due to some initial (before release) phase fluctuations [22] in the disordered BEC or due to interference of a few BECs separated by high potential barriers with no well-defined relative phase between them, as in either of these scenarios the fringe positions are not expected to be reproducible.

Numerical simulations based on the Gross-Pitaevskii equation $[11,21]$. have shown that such interference can occur when moderate disorder-induced density fluctuations cause different parts of a phase-coherent BEC to expand with different velocities and overlap. As $V_{D}$ is increased from 0 , the contrast of the interference is expected to increase, consistent with the data shown in Fig. 5 at small $V_{D}$. When $V_{D}$ becomes sufficiently large, however, the BEC fragments into multiple pieces, as is seen in the in situ image, Fig. 4(i). An array of randomly spaced condensates should not produce visible interference in TOF [23,24], again consistent with our observation of diminishing fringe contrast at high $V_{D}$ [Fig. 4(j)].

At high $V_{D}$, we observe completely inhibited transport, indicating a transition to an insulator. This was seen in previous transport experiments, where the nature of the insulator is inferred to be that of a fragmented BEC [8-10]. Our in situ images give the first direct observation of fragmentation of a highly disordered BEC. Because of the exponentially suppressed Josephson tunneling between fragments due to high potential barriers, the fragmented BEC is expected to have large phase fluctuations and no phase coherence [25]. This regime is analogous to a granular superconductor $[3,4]$, which is an insulator without global phase coherence.

Dipole oscillations are damped at moderate $V_{D}$, as shown in Fig. 3, and in agreement with previous observations [8]. Excitations such as solitons, vortices, and phase slips have been suggested [27] as possible mechanisms giving rise to damping. Our in situ as well as TOF data at moderate $V_{D}$ suggest a disordered BEC that is still connected (not yet fragmented) and phase coherent in order to give a reproducible interference pattern. Such a state may provide insight into the physics of "homogenous" disordered superconducting films, where there has been tremendous interest regarding the form of the order parameter, possibilities of metallic states, and the fate of phase coherence and phase stiffness [28] during the SIT [3,7]. Damped dipole oscillations were also recently studied for a BEC in a periodic potential [29] in the context of a possible "Bose metal" phase [7].

Strong repulsive interactions are believed to preclude Anderson localization (AL) $[11,25]$ in a BEC such as ours and those in previous experiments where the condensate healing length is small in comparison to $\sigma_{z}[8-11]$. Interactions, however, can be significantly reduced in ${ }^{7} \mathrm{Li}$ due to 
an extremely gradual zero crossing in the $B$ dependence of $a_{s}[15,16]$, creating more favorable conditions to observe AL. Such experiments are currently underway. A similar zero crossing has been identified in ${ }^{39} \mathrm{~K}$ [26].
Financial support of this work was provided by NSF, ONR, the Welch Foundation (Grant No. C-1133), and the Keck Foundation. We thank A. Aspect, L. Sanchez-Palencia, and T. A. Corcovilos for helpful discussions.
[1] P. W. Anderson, Phys. Rev. 109, 1492 (1958); P. A. Lee and T. V. Ramakrishnan, Rev. Mod. Phys. 57, 287 (1985).

[2] J. D. Reppy, J. Low Temp. Phys. 87, 205 (1992).

[3] A. M. Goldman and N. Markovic, Phys. Today 51(11), 39 (1998); Y. Dubi, Y. Meir, and Y. Avishai, Nature (London) 449, 876 (2007).

[4] I. S. Beloborodov, A. V. Lopatin, V. M. Vinokur, and K. B. Efetov, Rev. Mod. Phys. 79, 469 (2007).

[5] E. Granato and J. M. Kosterlitz, Phys. Rev. B 33, 6533 (1986); M. Y. Choi, J. S. Chung, and D. Stroud, ibid. 35, 1669 (1987).

[6] M. P. A. Fisher, Phys. Rev. Lett. 65, 923 (1990).

[7] P. Phillips and D. Dalidovich, Science 302, 243 (2003).

[8] J. E. Lye, L. Fallani, M. Modugno, D. S. Wiersma, C. Fort, and M. Inguscio, Phys. Rev. Lett. 95, 070401 (2005).

[9] C. Fort, L. Fallani, V. Guarrera, J. E. Lye, M. Modugno, D. S. Wiersma, and M. Inguscio, Phys. Rev. Lett. 95, 170410 (2005)

[10] D. Clément, A. F. Varon, M. Hugbart, J. A. Retter, P. Bouyer, L. Sanchez-Palencia, D. M. Gangardt, G. V. Shlyapnikov, and A. Aspect, Phys. Rev. Lett. 95, 170409 (2005); D. Clément et al., New J. Phys. 8, 165 (2006).

[11] T. Schulte, S. Drenkelforth, J. Kruse, W. Ertmer, J. Arlt, K. Sacha, J. Zakrzewski, and M. Lewenstein, Phys. Rev. Lett. 95, 170411 (2005).

[12] L. Fallani, J. E. Lye, V. Guarrera, C. Fort, and M. Inguscio, Phys. Rev. Lett. 98, 130404 (2007); J. E. Lye, L. Fallani, C. Fort, V. Guarrera, M. Modugno, D. S. Wiersma, and M. Inguscio, Phys. Rev. A 75, 061603(R) (2007).

[13] M. P. A. Fisher, P. B. Weichman, G. Grinstein, and D. S. Fisher, Phys. Rev. B 40, 546 (1989); W. Krauth, N. Trivedi, and D. Ceperley, Phys. Rev. Lett. 67, 2307 (1991).

[14] B. Damski, J. Zakrzewski, L. Santos, P. Zoller, and M. Lewenstein, Phys. Rev. Lett. 91, 080403 (2003); A. Sanpera, A. Kantian, L. Sanchez-Palencia, J. Zakrzewski, and M. Lewenstein, ibid. 93, 040401 (2004).

[15] K. E. Strecker, G. B. Partridge, A. G. Truscott, and R. G. Hulet, Nature (London) 417, 150 (2002).

[16] L. Khaykovich et al., Science 296, 1290 (2002).

[17] J. W. Goodman, Speckle Phenomena in Optics (Roberts, Greenwood Village, CO, 2007).

[18] We have checked that ramping on of the disordered potential does not produce significant heating by ramping the disordered potential back to zero to recover a pure condensate.

[19] C. C. Bradley, C. A. Sackett, and R. G. Hulet, Phys. Rev. Lett. 78, 985 (1997).

[20] The in situ images are taken immediately (within $5 \mu$ s) after the cloud is released from the optical trap.

[21] D. Clément, P. Bouyer, A. Aspect, and L. Sanchez-Palencia, preceding paper, Phys. Rev. A 77, 033631 (2008).

[22] S. Dettmer et al., Phys. Rev. Lett. 87, 160406 (2001).

[23] Z. Hadzibabic, S. Stock, B. Battelier, V. Bretin, and J. Dalibard, Phys. Rev. Lett. 93, 180403 (2004).

[24] L. Fallani, C. Fort, J. E. Lye, and M. Inguscio, Opt. Express 13, 4303 (2005).

[25] L. Sanchez-Palencia, D. Clement, P. Lugan, P. Bouyer, G. V. Shlyapnikov, and A. Aspect, Phys. Rev. Lett. 98, 210401 (2007); L. Sanchez-Palencia, Phys. Rev. A 74, 053625 (2006); P. Lugan, D. Clement, P. Bouyer, A. Aspect, M. Lewenstein, and L. Sanchez-Palencia, Phys. Rev. Lett. 98, 170403 (2007).

[26] G. Roati, M. Zaccanti, C. D’Errico, J. Catani, M. Modugno, A. Simoni, M. Inguscio, and G. Modugno, Phys. Rev. Lett. 99, 010403 (2007).

[27] T. Paul, P. Schlagheck, P. Leboeuf, and N. Pavloff, Phys. Rev. Lett. 98, 210602 (2007).

[28] P. W. Anderson, Basic Notions of Condensed Matter Physics (Westview, Boulder, CO, 1997).

[29] D. McKay, M. White, M. Pasienski, and B. DeMarco, e-print arXiv:0708.3074. 